Cite this: Phys. Chem. Chem. Phys., 2014, 16, 25684

Received 14th March 2014 , Accepted 14th April 2014

DOI: $10.1039 / c 4 c p 01111 b$

www.rsc.org/pccp

\title{
Influence of nanoparticle shape on charge transport and recombination in polymer/ nanocrystal solar cells $\dagger$
}

\author{
Zhe Li, $\ddagger^{*^{a}}$ Weiyuan Wang, ${ }^{a}$ Neil C. Greenham ${ }^{a}$ and Christopher R. McNeill*b
}

\begin{abstract}
A key consideration for the efficient operation of hybrid solar cells based upon conjugated polymers and inorganic semiconductor nanocrystals is charge transport in the nanocrystal phase. Here we report the results of a study into the charge transport kinetics of polymer/nanocrystal solar cells based on blends poly(3-hexylthiophene) (P3HT) with either CdSe nano-dots or CdSe nano-tetrapods. Transient photocurrent measurements reveal significant differences in the charge transport kinetics of nano-dot and nano-tetrapod hybrid cells, with the charge collection of the P3HT/CdSe nano-dot device severely limited by charge trapping. In comparison the nano-tetrapod cell exhibits significantly reduced charge trapping compared to the nano-dot cell accounting for the improved fill-factor and overall device efficiency. Transient photovoltage measurements have also been employed that demonstrate slower recombination rates in the $\mathrm{P} 3 \mathrm{HT} / \mathrm{CdSe}$ tetrapod device compared to the P3HT/CdSe dot device. These observations directly identify nanoparticle shape as a critical factor influencing the charge transport and hence recombination in this benchmark hybrid system, confirming the hypothesis that the use of tetrapods improves device performance through an improvement in electron transport in the nanocrystal phase.
\end{abstract}

\section{Introduction}

Organic bulk heterojunction (BHJ) solar cells have demonstrated significant improvements in both operating lifetime ( $\sim 7$ years) and power conversion efficiency (over 9\%) owing to rapid advances in both materials and device design. ${ }^{1,2}$ Hybrid solar cells based on blends of conjugated polymers and inorganic semiconducting nanocrystals are an important class of organic $\mathrm{BHJ}$ solar cell with power conversion efficiencies of over $4 \%$ recently reported. ${ }^{3}$ This type of solar cell benefits from the tuneable physical properties of inorganic semiconducting nanocrystals; varying nanoparticle size and shape during synthesis provides a flexible yet controllable way to improve device performance. ${ }^{4}$

Hybrid BHJ solar cells are typically based on blends of conjugated polymers and inorganic quantum dots. ${ }^{5,6}$ However, despite the suitable energy levels of these nanoparticles enabling them to act as efficient electron acceptors in blends with

\footnotetext{
${ }^{a}$ Cavendish Laboratory, University of Cambridge, JJ Thomson Ave, Cambridge, CB3 OHE, UK

${ }^{b}$ Department of Materials Engineering, Monash University, Clayton, Victoria 3800, Australia.E-mail: christopher.mcneill@monash.edu

$\dagger$ Electronic supplementary information (ESI) available. See DOI: 10.1039/ c4cp01111b

\# Current address: Department of Chemistry, Imperial College London, London SW7 2AZ, UK. E-mail: zhe.li@imperial.ac.uk
}

conjugated polymers, ${ }^{7}$ early devices typically yielded relatively low efficiencies, which was attributed to poor electron transport in the nanoparticle phase. ${ }^{5}$ Indeed, limited by inefficient nanoparticle-to-nanoparticle hops, poor electron transport in the nanoparticle phase in hybrid solar cells is a key consideration that significantly limits the device performance of this type of solar cell. ${ }^{8,9} \mathrm{~A}$ number of morphological and energetic factors, such as "dead ends" and interfacial trap states have been proposed to be major detrimental factors that lead to severe charge trapping. ${ }^{10}$ To date, significant research effort has been dedicated to improving the charge transport in hybrid polymer/nanoparticle solar cells. A successful approach to improve the efficiency of polymer/nanocrystal solar cells has been to replace nano-dots with nano-rods, the hypothesis being that the elongated physical shape of nano-rods facilitates enhanced electron transport due to reduced number of interparticle hops, thereby profoundly reducing the possibility of non-geminate recombination during charge transport and hence improving power conversion efficiency. ${ }^{11}$ However, recent electron tomography studies have demonstrated that when incorporated into blend films, most of the nano-rods are oriented parallel to the plane of the film, which still limits charge transport of these devices in the direction perpendicular to the film plane. ${ }^{12}$ With the aim of further enhancing charge transport, nano-rods were replaced by nano-tetrapods or hyper-branched nanoparticles, 
since the three dimensional shape of these nanoparticles makes them impossible to locate parallel within the film. Devices based on tetrapods or hyper-branched nanoparticles demonstrate further improved efficiency compared to rodbased hybrid solar cells. ${ }^{13-16}$

However, while it is generally accepted that nanoparticle shape plays an important role in influencing charge transport and hence device efficiency, ${ }^{17}$ only limited studies have systematically investigated the charge transport and recombination in hybrid solar cells. In 2003, Huynh and coworkers investigated the charge transport in hybrid polymer-nanorods solar cells through the modelling of the current-voltage characteristics, and reported that the device physics of this type of solar cells can be described using a Shockley-type approach accounting for shunt/series resistance and space-charge effects. ${ }^{18}$ Later, Dayal and coworkers performed electrodeless flash photolysis time-resolved microwave conductivity (FP TRMC) measurements to show that nanorods and tetrapods devices benefit from a higher aspect ratio that results in increased carrier lifetime and device efficiency compared to nanodots devices. ${ }^{19}$ However, these techniques are limited to only being able to provide indirect information regarding charge transport in inorganic hybrid blends and in particular how charge transport impacts device performance.

As a powerful technique to study the fate of separated charge carriers in organic solar cells, transient photocurrent and transient photovoltage techniques are particularly suited to directly investigating charge transport and recombination dynamics. ${ }^{8,20,21}$ Herein, we report a transient photocurrent and transient photovoltage study on the operation of the benchmark P3HT/ CdSe nanoparticle hybrid solar system, examining the influence of nanoparticle shape upon the operational mechanisms of this system. P3HT/CdSe nano-dots and P3HT/CdSe nanotetrapods blends were selected for a comparative study, employing the same batch of $\mathrm{P} 3 \mathrm{HT}$ and following the same device fabrication procedure. The CdSe nano-dots and nano-tetrapods were synthesized in-house with the same quantum confinement energy, ensuring a direct side-by-side comparison. Through analysis of the transient photocurrent and transient photovoltage studies, significantly reduced charge trapping and hence trap-mediated recombination is revealed in the $\mathrm{P} 3 \mathrm{HT} /$ CdSe tetrapod device compared to the P3HT/CdSe dot device. These measurements therefore provide direct experimental evidence for the significant influence of the nanoparticle shape upon the charge transport and hence overall device performance of hybrid solar cells.

\section{Results and discussion}

\section{Steady state device characterisation}

Fig. 1(a-c) presents the steady state device characteristics of the $\mathrm{P} 3 \mathrm{HT} / \mathrm{CdSe}$ dot and P3HT/CdSe tetrapod devices. The CdSe dots used for the P3HT/CdSe dot device fabrication had a diameter of $\sim 6.4 \mathrm{~nm}$, while the CdSe tetrapods used for the P3HT/CdSe tetrapod device fabrication had a leg length of $\sim 20 \mathrm{~nm}$ and a leg diameter of $\sim 6 \mathrm{~nm}$ (see Fig. S1 in ESI $\dagger$ for TEM images). The EQE spectra of both devices, Fig. 1(a),
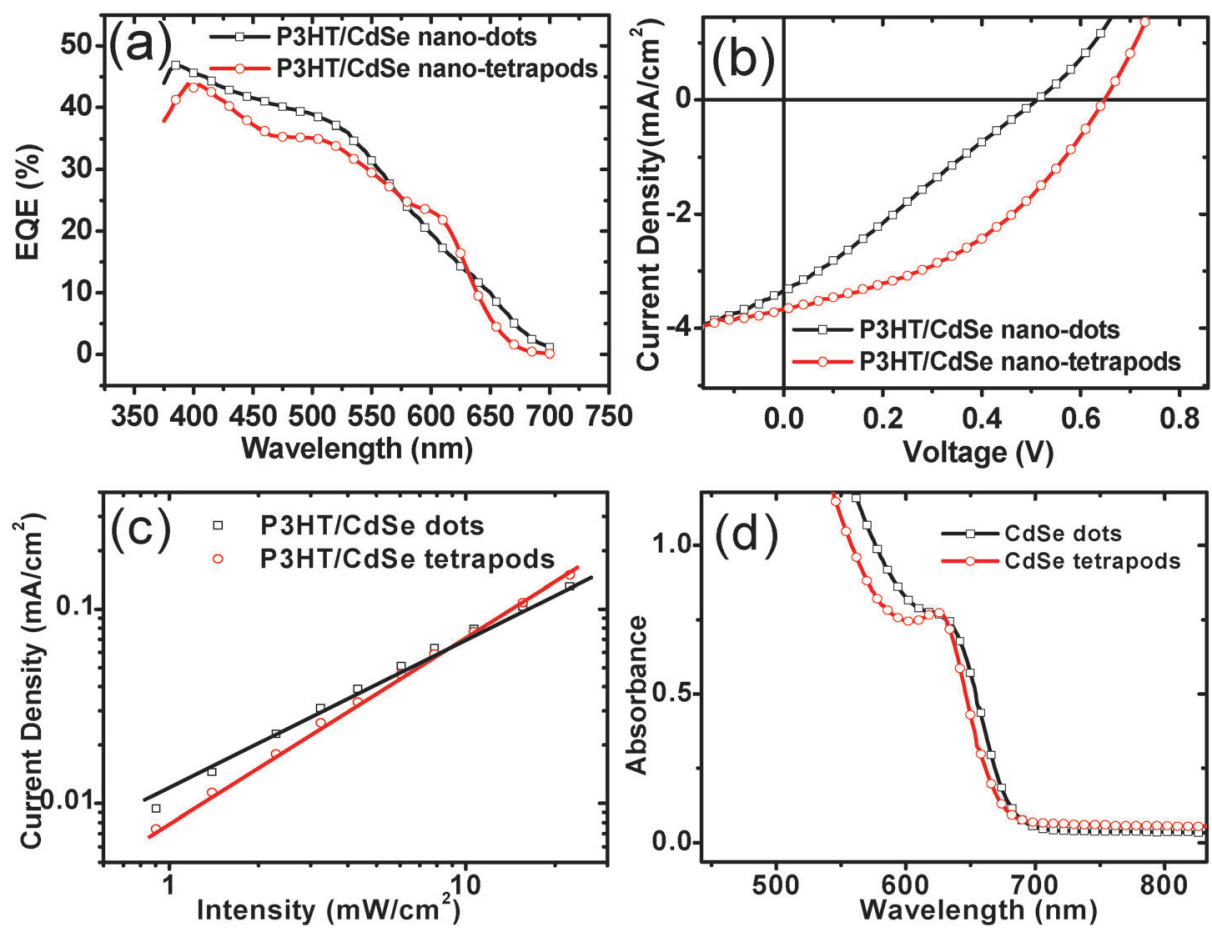

Fig. 1 (a) EQE, (b) current-voltage characteristics under $100 \mathrm{~mW} \mathrm{~cm}{ }^{-2} \mathrm{AM} 1.5 \mathrm{G}$ illumination and (c) steady state light intensity dependence of the shortcircuit photocurrent under monochromatic illumination at $525 \mathrm{~nm}$ for the P3HT/CdSe dot (squares) and P3HT/CdSe tetrapod (circles) devices. (d) Absorption spectra of the synthesised CdSe nanoparticles in solution. 
have a similar shape in the blue to green region of the solar spectrum where the nanoparticles absorb, consistent with a similar degree of quantum confinement which is determined by the similar smallest dimension of the nanoparticles $(\sim 6 \mathrm{~nm}$ for both systems). This observation is also consistent with the similar absorption peak position of the two types of nanoparticles $(\sim 633 \mathrm{~nm}$ for CdSe dots $v s . \sim 626 \mathrm{~nm}$ for CdSe tetrapods), with their absorption spectra provided in Fig. 1(d). The more pronounced shoulder at $\sim 600 \mathrm{~nm}$ for the P3HT/CdSe tetrapod device is indicative of enhanced P3HT crystallinity that should also facilitate improved transport of holes in this device. $^{22}$ Examining the current-voltage characteristics under AM1.5G simulated solar illumination, Fig. 1(b), the P3HT/CdSe tetrapod device exhibits a slightly higher $J_{\mathrm{SC}}\left(3.7 \mathrm{~mA} \mathrm{~cm} \mathrm{~cm}^{-2} v s\right.$. $3.3 \mathrm{~mA} \mathrm{~cm}^{-2}$ ) despite a slightly lower peak EQE than the P3HT/ CdSe dot device. The overall performance of the P3HT/CdSe tetrapod device benefits from a significantly enhanced $V_{\mathrm{OC}}$ (0.65 V vs. $0.51 \mathrm{~V})$ and $\mathrm{FF}$ (0.43 vs. 0.27$)$, leading to a superior power conversion efficiency $(1.0 \%$ vs. $0.45 \%)$ compared to the P3HT/CdSe dot device, consistent with previous studies. ${ }^{13,19}$ Fig. 1(c) presents the steady-state light-intensity dependence of $J_{\text {SC }}$ of the two devices. The P3HT/CdSe tetrapod device exhibits a near-linear dependence of $J_{\mathrm{SC}}$ on light intensity with coefficient of $\alpha=0.94$ corresponding to the power law fit $J_{\mathrm{SC}} \propto I^{\alpha}$ where $I$ is the light intensity. The P3HT/CdSe dot device in contrast shows a more non-linear dependence of $J_{\mathrm{SC}}$ on light intensity with coefficient of $\alpha=0.82$, indicative of more pronounced higher order recombination losses. Notably the $J_{\mathrm{SC}}$ of the P3HT/CdSe tetrapod device exceeds that of the P3HT/CdSe dot device at higher light intensities, which explains the observation of a lower EQE (measured under low light intensity) but higher $J_{\mathrm{SC}}$ (measured under AM 1.5 solar illumination) of the P3HT/CdSe tetrapod device compared to the P3HT/CdSe dot device.

\section{Short-circuit transient photocurrent measurements}

Fig. 2 presents the short-circuit photocurrent transients of the P3HT/CdSe dot and P3HT/CdSe tetrapod devices. The shortcircuit photocurrent transients of the devices in response to $200 \mu$ s square light pulses were recorded as a function of pulsed light intensity (Fig. 2a and c), with normalised traces plotted in Fig. 2(c and d). A steady current is reached by $t=200 \mu \mathrm{s}$, with the light-intensity dependence of the photocurrent matching that of the steady-state characterisation indicating that the devices have indeed reached steady state. The transient shortcircuit photocurrent traces of both devices exhibit an initial fast rise, to about $50 \%$ of the steady-state value, followed by a slower rise to the steady-state value. Previous modelling of photocurrent dynamics ${ }^{23}$ have shown that with an imbalance in electron-hole mobilities, the fast rise to $\sim 50 \%$ of the steadystate signal can be attributed to the dynamics of the faster carrier and the subsequent slow rise to the dynamics of the slower carrier. Since P3HT/PCBM devices exhibit a rise time of only $3 \mu \mathrm{s},{ }^{8}$ the slower second rise time observed here is entirely consistent with slower charge transport processes in the nanocrystal phase compared to the polymer phase. Interesting differences are seen in the intensity dependence of the shape of the short-circuit photocurrent traces of the P3HT/CdSe dot
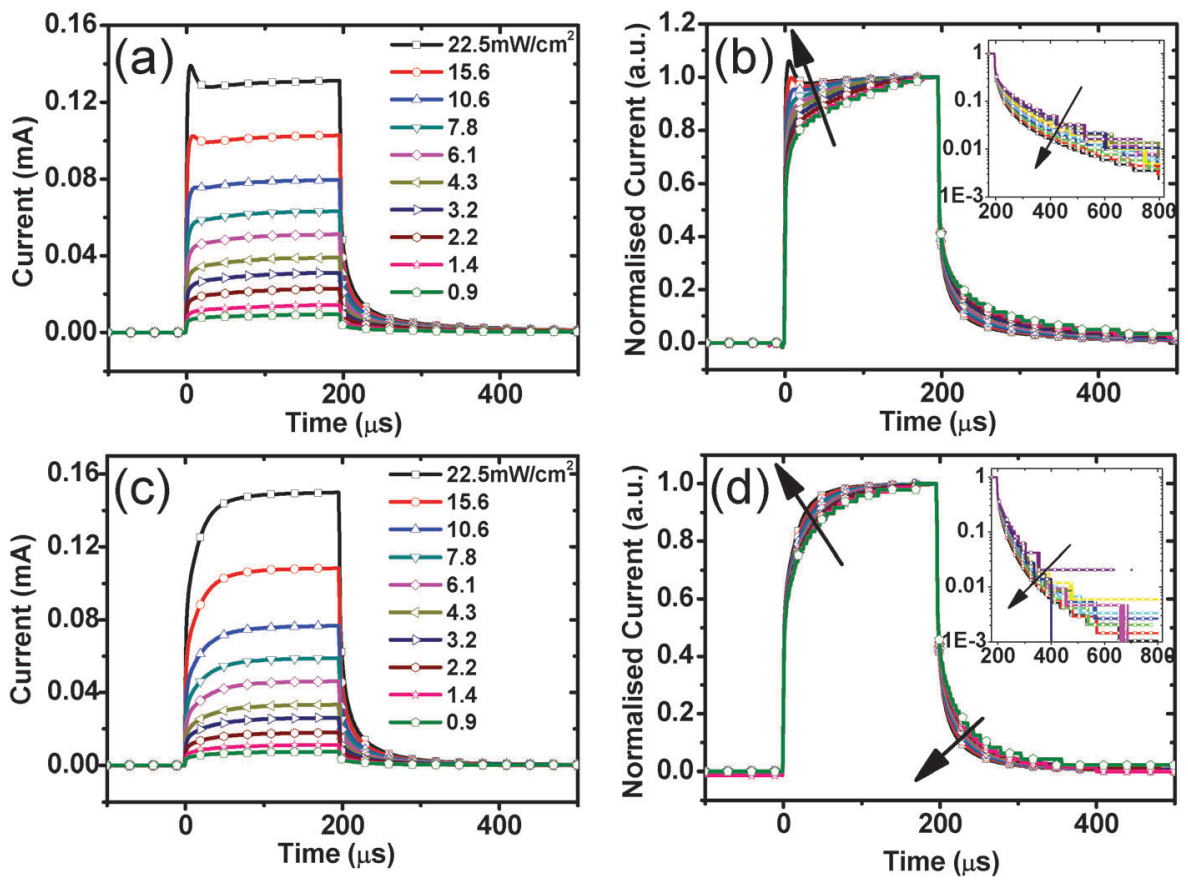

Fig. 2 Transient short-circuit photocurrent of the P3HT/CdSe dot device, (a, b), and the P3HT/CdSe tetrapod device, (c, d), in response to $200 \mu$ s square pulsed illumination with a wavelength of $525 \mathrm{~nm}$ at various intensities. $(\mathrm{a}, \mathrm{c})$ present the raw data while $(\mathrm{b}, \mathrm{d})$ present the transient photocurrent curves normalised to the photocurrent value at $200 \mu \mathrm{s}$. The insets of figure parts (b) and (d) highlight the decay kinetics of the short-circuit photocurrent transients after normalisation. 

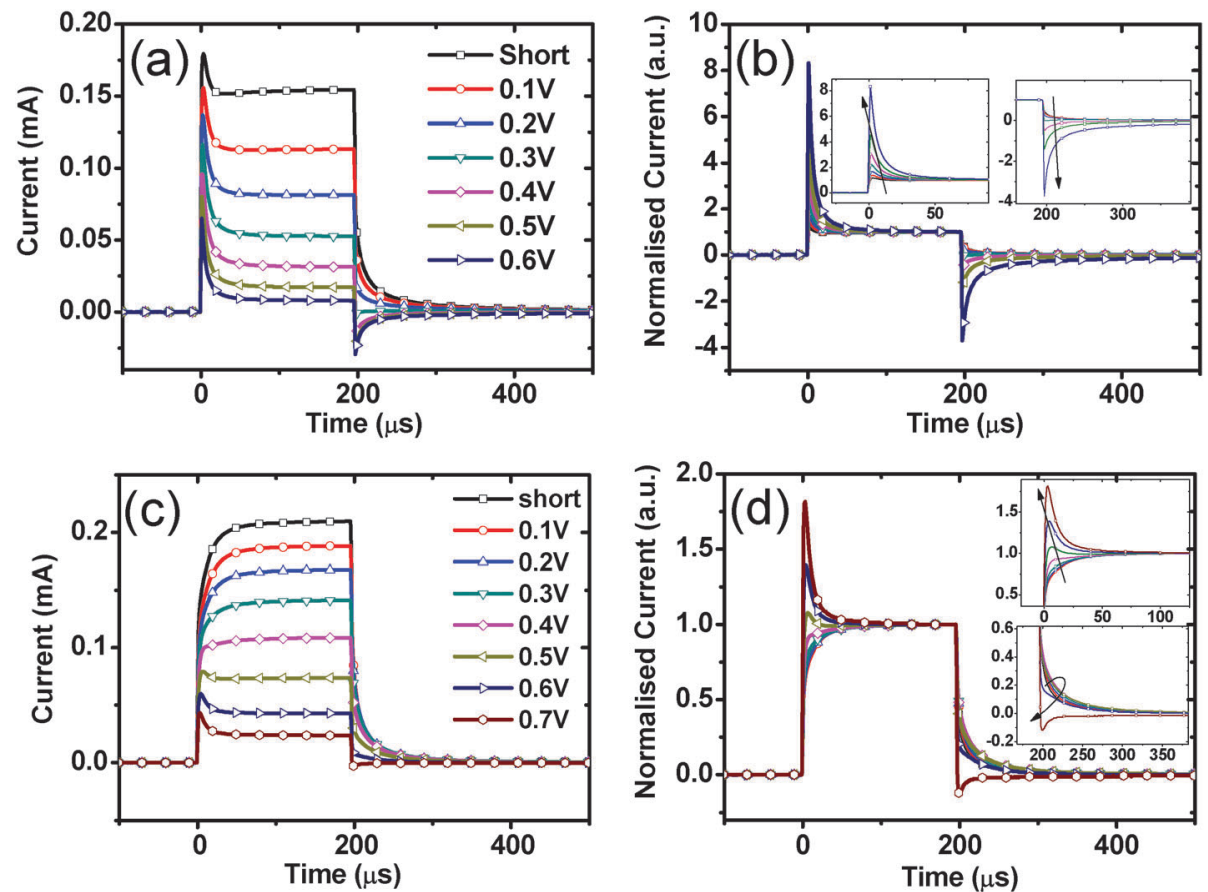

Fig. 3 Transient photocurrent response of the P3HT/CdSe dot (a, b) and P3HT/CdSe tetrapod (c, d) devices to $200 \mu$ s square-pulse illumination as a function of applied bias voltage. Figure parts $(a, c)$ plot un-normalised traces whereas $(b, d)$ plot normalised traces. Insets of figure parts $(b, d)$ highlight the rise and decay kinetics of the voltage-dependent transients after normalisation. Pulse intensity is $\sim 32 \mathrm{~mW} \mathrm{~cm}^{-2}$

device (Fig. 2a and b) compared to the tetrapod device (Fig. 2c and d). With increasing light intensity, an initial transient peak (5-10 $\mu$ s after turn-on) appears in the turn-on dynamics of the nano-dot device. Such a photocurrent overshoot has been previously interpreted in terms of severe trap-mediated recombination in conjunction with a slow detrapping rate in this system. $^{8,20,23}$ As reproduced by numerical simulations, the overshoot occurs since the build-up of trapped charge occurs on a slower timescale to the initial extraction of mobile carriers, with the rate of recombination increasing with increased charge density. ${ }^{23}$ While both the nano-dot and nano-tetrapod devices exhibit a slow rise, consistent with the slower transport kinetics of electrons in the nanocrystal phase, only the nanodot device exhibits the photocurrent overshoot at short-circuit pointing to enhanced trap-mediated recombination in the nano-dot device at short circuit. Examining the decay kinetics of the photocurrent transients (see insets of figure part b, d), faster charge extraction is observed in the P3HT/CdSe tetrapod device compared to the P3HT/CdSe dot device $(\sim 340 \mu$ s for current to decay to $1 \%$ of its steady state value for the P3HT/ CdSe tetrapod device compared to $\sim 470 \mu$ s for the P3HT/CdSe dot device). These observations are indicative of improved charge extraction due to reduced charge trapping in the P3HT/CdSe tetrapod system compared to the P3HT/CdSe dot system.

\section{Voltage-dependent transient photocurrent measurements}

Fig. 3 presents the voltage-dependent transient photocurrent response of the $\mathrm{P} 3 \mathrm{HT} / \mathrm{CdSe}$ dot and $\mathrm{P} 3 \mathrm{HT} / \mathrm{CdSe}$ tetrapod devices at a constant pulse intensity of $\sim 32 \mathrm{~mW} \mathrm{~cm}^{-2}$. The magnitude of the photocurrent overshoot in the P3HT/CdSe dot device, Fig. 3(a and b), increases as the working voltage is swept towards open circuit, consistent with previous observations. ${ }^{8}$ Our previous studies have quantitatively reproduced this feature through numerical simulations accounting for two major loss mechanisms inside the device, namely the Shockely-Read-Hall (SRH) recombination and bimolecular recombination. ${ }^{24}$ During earlier times of illumination, trapped charges (especially electrons) are rapidly built up, favouring SRH recombination between trapped electrons and free holes, becoming more severe at higher applied bias voltages (lower internal electric fields). After steady state is reached where all traps are filled up and maintained at dynamic equilibrium, bimolecular recombination becomes more important and competes with charge extraction. The P3HT/CdSe tetrapod device (Fig. 3c) in contrast exhibits smooth rising dynamics of the photocurrent transients at lower applied bias voltages (from short circuit to $0.4 \mathrm{~V}$ ), with a photocurrent overshoot only visible for working voltages higher than $0.5 \mathrm{~V}$. This lack of any transient peak after turn on around shortcircuit can be interpreted in terms of a lower density of charge traps or that SRH/trap-mediated recombination is inhibited by the higher internal electric field at lower applied bias voltages. Examining the decay kinetics of the un-normalised voltagedependent photocurrent transients (see Fig. S2 in ESI $\dagger$ ), the photocurrent traces overlap for the applied bias voltages from $0 \mathrm{~V}$ to $0.4 \mathrm{~V}$, suggesting a similar equilibrium trapped charge density at this light intensity that is independent of voltage. Therefore, the absence of the initial transient is more likely due to inhibited SRH/trap-mediated recombination rather than reduced density of charge traps at lower applied bias voltages 

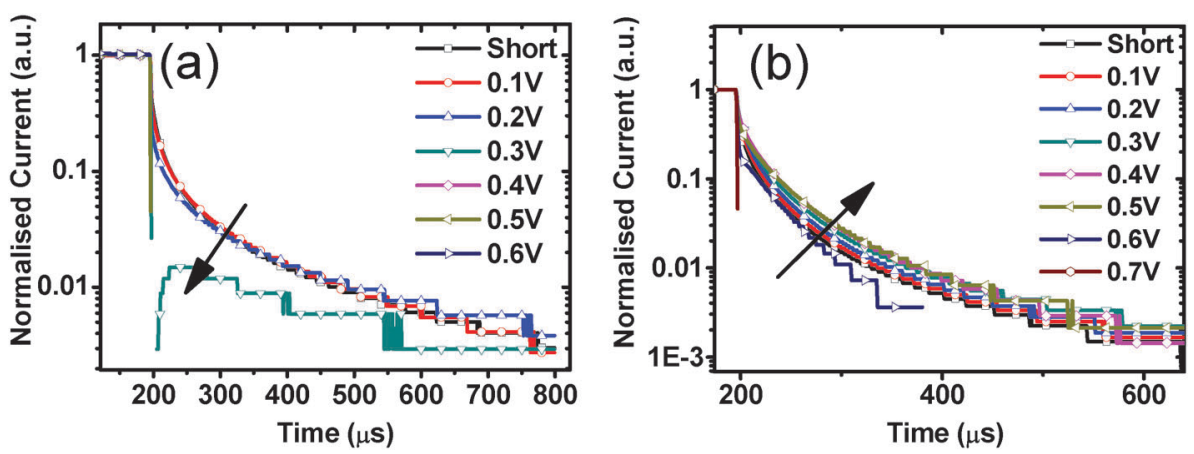

Fig. 4 Normalised voltage-dependent photocurrent decay transients for (a) the P3HT/CdSe dot device and (b) the P3HT/CdSe tetrapod device on a log scale.

for the P3HT/CdSe tetrapod device. Notably the relative magnitude of the initial transient peak to the steady-state photocurrent is much higher for the P3HT/CdSe dot device compared to the P3HT/CdSe tetrapod device ( $\sim 8$ times for the dot device compared to $\sim 1.5$ times for the tetrapod device at an applied bias voltage of $0.6 \mathrm{~V}$, see insets of figure parts $\mathrm{b}$ and d), an indication of more significant SRH/trap-mediated recombination losses in the $\mathrm{P} 3 \mathrm{HT} / \mathrm{CdSe}$ dot device compared to the $\mathrm{P} 3 \mathrm{HT} / \mathrm{CdSe}$ tetrapod device. After turn-off, the P3HT/CdSe dot device exhibits a negative photocurrent transient, attributed to the back injection of holes to neutralise the trapped electrons that are not readily extracted. ${ }^{8,24}$ In contrast, no back injection of charge is observed for the P3HT/CdSe tetrapod device for applied bias voltages less than $V_{\mathrm{OC}}(\sim 0.65 \mathrm{~V})$. This observation indicates that the extraction of electrons from the nanocrystal phase in the tetrapod device is more efficient than in the nano-dot device, with a lower density of deeply trapped charges.

Fig. 4 presents the decay kinetics of the voltage-dependent photocurrent transients for the applied voltages where the photocurrent remains positive after turn-off. No pronounced slowing of the photocurrent decay transients is observed as the voltage is moved from short circuit to open circuit for both devices, indicating that charge transport in both systems is largely dispersive. (In the case of non-dispersive charge transport, voltage dependent photocurrent decay transients are expected since the average charge extraction time is determined by the drift of free charge carriers and varies with electric filed. $\left.{ }^{8}\right)$ For the P3HT/CdSe dot device, Fig. 4(a), the turn-off dynamics actually quicken as the applied voltage is moved from short circuit to $V_{\mathrm{OC}}$, indicating that recombination dominates charge extraction even for applied voltages close to short circuit. For the P3HT/CdSe tetrapod device, however, a mild retarding of the photocurrent tail is observed as the working voltage is moved towards open circuit, followed by a rapid quickening of the decay transients for voltages greater than $0.5 \mathrm{~V}$. Considering the three dimensional elongated shape of the CdSe nano-tetrapods, the mild broadening of the decay kinetics of the photocurrent transients close to short circuit is most likely due to reduced number of times a charge is trapped and detrapped before being collected, hence providing evidence for improved charge transport and reduced charge recombination along the direction of the internal electric field (perpendicular to the film plane) in the P3HT/CdSe tetrapod device. The quickening of the decay transients at higher applied bias voltages (higher than $0.5 \mathrm{~V}$ ) can be explained by charge recombination dominating over charge extraction at lower electric field. Although geminate recombination may also contribute, the appearance of a transient peak after turn on for $V=0.5 \mathrm{~V}$ symptomatic of SRH/trap-mediated recombination indicates that charge trapping is important in facilitating charge recombination in this part of the $J V$ curve. Finally, the fundamental differences in the voltage-dependent decay kinetics of the photocurrent transients of the P3HT/CdSe dot and tetrapod devices point to significantly different detrapping dynamics in these two systems: compared to the P3HT/CdSe dot device that exhibits a strong voltage dependence of the decay kinetics of the transient photocurrent traces, the relatively voltage insensitive photocurrent decay transients of the P3HT/CdSe tetrapod device are indicative of a less voltage-dependent detrapping rate. This phenomenon suggests that compared to the $\mathrm{P} 3 \mathrm{HT} /$ CdSe dot device which relies on a strong internal electric field to detrap the trapped charge carriers (particularly electrons), the detrapping process is much more efficient in the $\mathrm{P} 3 \mathrm{HT} /$ CdSe tetrapod system and is much less field-assisted. This observation is also consistent with a much higher fill factor due to more efficient, less field-dependent charge extraction of the P3HT/CdSe tetrapod system compared to the P3HT/CdSe dot system.

\section{Charge extraction studies}

Fig. 5(a) plots the extracted charge as a function of light intensity at short circuit on a log scale. The amount of extracted charge is determined by integrating the decay kinetics of the photocurrent transients after turn-off. In the absence of charge trapping and severe non-geminate recombination, the amount of extracted charge is a measure of the device charge density and scales linearly with light intensity. For both the P3HT/CdSe dot and P3HT/CdSe tetrapod devices, a non-linear relationship is observed between the extracted charge and light intensity, consistent with charge extraction limited by trap-mediated recombination. Notably, despite more severe non-geminate recombination as evidenced by a more non-linear relationship of the current $v s$. intensity curve, a larger amount of charge is 

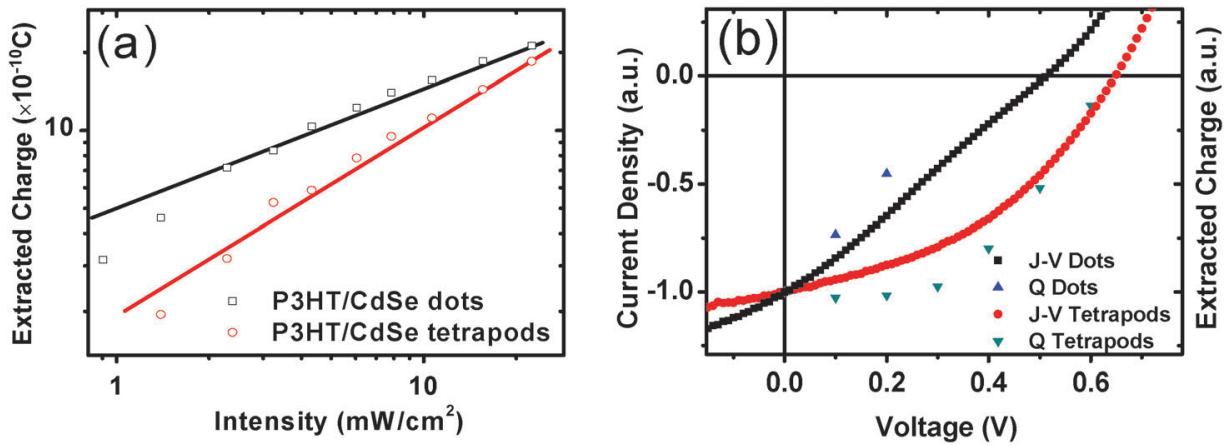

Fig. 5 (a) Plot of extracted charge after turn-off vs. illumination intensity at short circuit for the P3HT/CdSe dot and P3HT/CdSe tetrapod devices. The solid lines represent the linear fits of the extracted charge vs. intensity. (b) Normalised plot of extracted charge vs. voltage at $32 \mathrm{~mW} \mathrm{~cm}^{-2}$ monochromatic illumination for the P3HT/CdSe dot and P3HT/CdSe tetrapod devices. Normalised J-V characteristics are also plotted for comparison.

extracted out of the $\mathrm{P} 3 \mathrm{HT} / \mathrm{CdSe}$ dot device compared to the P3HT/CdSe tetrapod device, indicating that at short-circuit the P3HT/CdSe dot device is in general operating at a much higher charge density than the tetrapod device. Fig. 5(b) plots the extracted charge as a function of applied bias voltage. Both the P3HT/CdSe dot and P3HT/CdSe tetrapod device exhibit a general decrease in extracted charge with increasing applied bias voltage, suggesting that charge extraction for both systems is limited by charge trapping (for a trap free system the device is able to sustain an increase in charge density due to slower sweep-out time of the charge carriers as observed for the P3HT/ PCBM system ${ }^{8}$ ). For the P3HT/CdSe dot device, the extracted charge decreases monotonically as the working voltage is moved toward open circuit and follows the same shape as the current-voltage characteristics of this device. These features are a strong indication that charge trapping is limiting charge extraction from short circuit to open circuit for the $\mathrm{P} 3 \mathrm{HT} / \mathrm{CdSe}$ dots system. In contrast, for the P3HT/CdSe tetrapod device, the extracted charge remains constant from short circuit to $\sim 0.3 \mathrm{~V}$ (the extracted charge decreases by only $1 \%$ from $0 \mathrm{~V}$ to $0.3 \mathrm{~V}$ ), followed by a sharp decrease in extracted charge with further increasing applied bias voltage. This observation reveals relatively field-independent charge extraction with significantly reduced charge trapping features at lower applied bias voltages in the P3HT/CdSe tetrapod system compared to the P3HT/CdSe dot system. Notably, for the P3HT/CdSe tetrapod device, the appearance of the initial transient peak coincides with the voltage at which the amount of extracted charge drops significantly $(\sim 0.4 \mathrm{~V})$, which also corresponds to the maximum power point of the $J-V$ characteristics. Thus SRH/trap-mediated recombination is still an important mechanism for the $\mathrm{P} 3 \mathrm{HT} /$ CdSe tetrapod device, with charge extraction limited by charge trapping at applied bias voltages close to $V_{\mathrm{OC}}$. Based on these observations, it follows that charge extraction in the $\mathrm{P} 3 \mathrm{HT} / \mathrm{CdSe}$ tetrapod device is less limited by charge trapping at lower applied bias voltages, with most of these trapped charge carriers finally detrapped and collected as photocurrent. With further increasing applied bias voltage, however, SRH/trapmediated recombination begins to play an important role and compete with charge extraction. In contrast, charge extraction is severely limited by charge trapping from short-circuit to open-circuit conditions for the P3HT/CdSe dot device.

\section{Transient photovoltage measurements}

Fig. 6(a and b) plot the photovoltage decay transients as a function of background light intensity of the P3HT/CdSe dot and P3HT/CdSe tetrapod devices. Photovoltage transients were recorded by measuring the voltage decay of the devices held at open circuit under continuous illumination following a small perturbative light pulse. A systematic quickening of the photovoltage decay transients is observed with increasing background illumination intensity for both devices, an indication of increased non-geminate recombination at higher light intensities. Comparing the photovoltage decay transients in figure parts (a) and (b), significantly slower photovoltage decay kinetics are seen for the P3HT/CdSe tetrapod device compared to the $\mathrm{P} 3 \mathrm{HT} / \mathrm{CdSe}$ dot device (for example, the time taken to decay to $1 \%$ of the initial photovoltage is $\sim 2.5 \mathrm{~ms}$ for the dot device compared to $\sim 8 \mathrm{~ms}$ for the tetrapod device at a light intensity of $4.8 \mathrm{~mW} \mathrm{~cm} \mathrm{~m}^{-2}$ ), indicating a much lower non-geminate recombination rate and hence a much longer charge carrier lifetime. The photovoltage decay kinetics of the P3HT/CdSe dot device can be fitted with a tri-exponential term expressed as $\delta V=A_{1} \exp \left(-t / T_{1}\right)+A_{2} \exp \left(-t / T_{2}\right)+A_{3} \exp \left(-t / T_{3}\right)$, where $A_{n}$ are the weighting parameters and $T_{n}$ are the time constants for each exponential decay term, consistent with previous studies. ${ }^{8}$ In contrast, the decay transients of the P3HT/CdSe tetrapod device can be well-fitted with a bi-exponential fit expressed as $\delta V=A_{1} \exp \left(-t / T_{1}\right)+A_{2} \exp \left(-t / T_{2}\right)$, consistent with the more linear decay of the photovoltage transients shown in Fig. 6(b). The fact that the transient photovoltage decay requires a multiexponential fit suggests that there are noninteracting populations of carriers, each contributing to shifting the Fermi level, but each only able to decay by a different route. Assigning these decay channels is not straightforward. It is plausible that two decay channels as exhibited by the P3HT/CdSe tetrapods system may be ascribed to Langvin recombination and SRH/trap-mediated recombination. The additional decay channel as exhibited by the P3HT/CdSe dots system may be due to a secondary trap with different characteristic trap energy (for example bulk single 

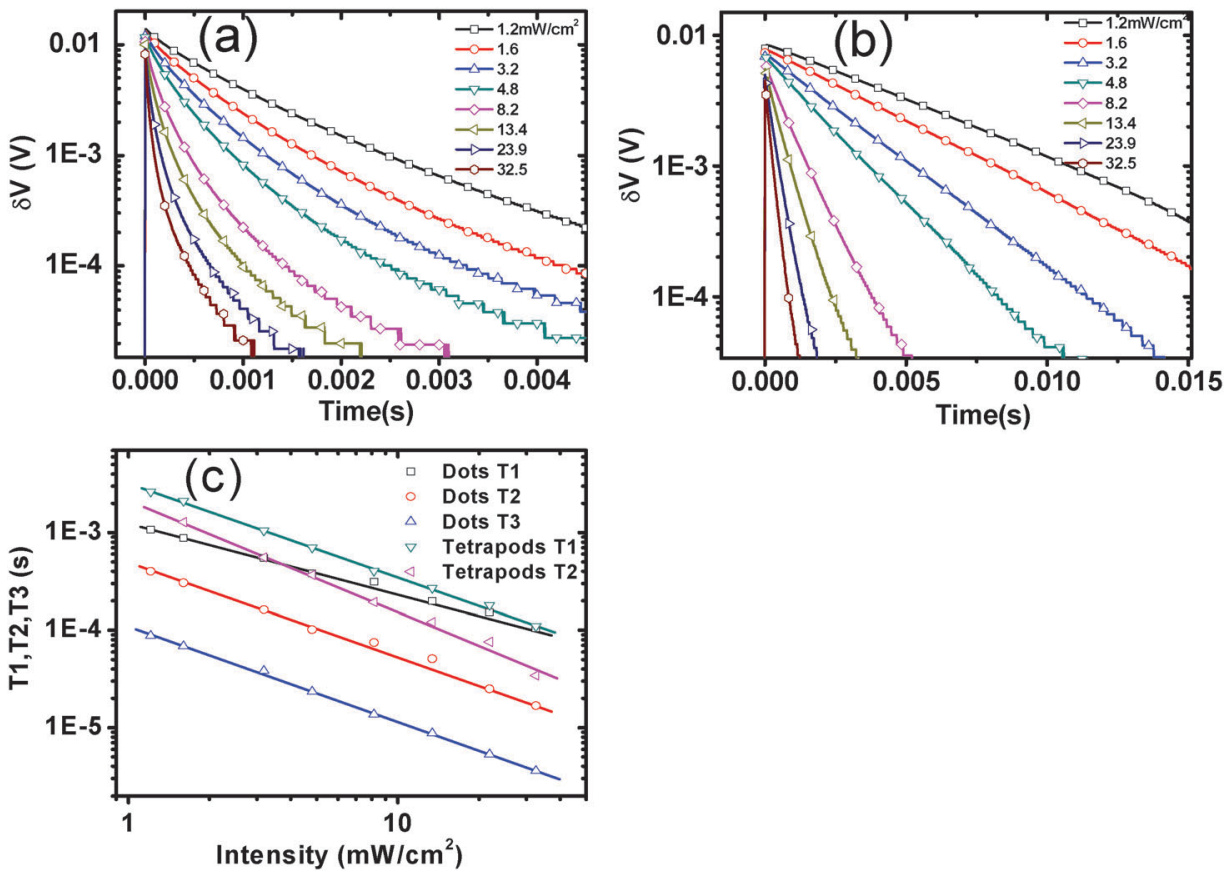

Fig. 6 Photovoltage decay transients plotted as a function of the white background constant illumination intensity for (a) the P3HT/CdSe dot device and (b) the P3HT/CdSe tetrapod device. Data is obtained by plotting the photovoltage transients on a log scale after subtraction of the background voltage. Figure part (c) plots the decay time constants with their linear fit as a function of background light intensity on a log-log scale.

nanoparticle trapping compared to surface trapping). The time constants $T_{n}$ are plotted in Fig. 6(c), where both the P3HT/CdSe dot and P3HT/CdSe tetrapod devices have time constants that decrease linearly with the logarithm of light intensity. At a background light intensity of $32.5 \mathrm{~mW} \mathrm{~cm} \mathrm{~cm}^{-2}$ that is close to AM1.5G solar illumination, the P3HT/CdSe dot device has time constants of $T_{1}=3.6 \mu \mathrm{s}, T_{2}=16.9 \mu \mathrm{s}$, and $T_{3}=95.5 \mu \mathrm{s}$, while the P3HT/CdSe tetrapod device has $T_{1}$ and $T_{2}$ values of $37.2 \mu \mathrm{s}$ and $113 \mu \mathrm{s}$ respectively. Notably the fastest decay channel (most likely assigned to Langvin recombination considering the fast recombination rate of free electrons and holes) has a charge carrier lifetime that is one order of magnitude higher in the P3HT/CdSe tetrapod system compared to the P3HT/CdSe dot system across the light intensity range investigated, consistent with reduced recombination losses of charge carriers under operating conditions of this system. These observations are indicative of reduced non-geminate recombination channels of separated charge carriers with a much longer charge carrier lifetime at open circuit in the P3HT/CdSe tetrapod device compared to the $\mathrm{P} 3 \mathrm{HT} / \mathrm{CdSe}$ dot device.

While significant charge trapping features are identified for the P3HT/CdSe dot and P3HT/CdSe tetrapod systems, the origin of charge trapping in both systems is not obvious. There are a number of factors that are potentially responsible for the reduced charge trapping in the $\mathrm{P} 3 \mathrm{HT} / \mathrm{CdSe}$ tetrapod system. First, considering the significantly larger volume of CdSe tetrapods compared to CdSe dots (the volume of a CdSe nano-dot with a diameter of $6 \mathrm{~nm}$ is less than $1 / 10$ of a CdSe tetrapod with leg length of $20 \mathrm{~nm}$ and diameter of $6 \mathrm{~nm}$ ), the number density of CdSe nano-dots is ten times more than that of CdSe tetrapods.
Therefore, more efficient electron transport due to a reduced number of nanoparticle to nanoparticle transport events is likely to be an important reason behind the improved charge transport for the P3HT/CdSe tetrapod device. Furthermore, for an un-optimised nano-morphology, a lower number density of CdSe nanoparticles may result in isolated CdSe nanoparticles throughout the bulk film. Since "dead ends" and isolated nanoparticles have been considered as a potential source for morphological charge trapping in the polymer/nanocrystal system, ${ }^{25}$ enhanced charge transport due to reduced trap density may also be responsible for the less severe charge trapping features in the P3HT/CdSe tetrapod device. Second, the mild voltage dependence of the photocurrent decay transients of the P3HT/CdSe tetrapods system suggests that charge transport is enhanced especially along the direction of the internal electric field, which may be explained by the enhanced electron transport perpendicular to the film plane due to the three-dimensional shape of the CdSe tetrapods compared to the zero-dimensional nano-dots. Notably the CdSe nano-tetrapods employed in the P3HT/CdSe tetrapod device typically have a leg length of more than $20 \mathrm{~nm}$, comparable to the film thickness of the device, which means much better connected pathways for electron transport in the P3HT/CdSe tetrapod system compared to the P3HT/CdSe dot system. Third, reduced trap density owing to reduced surface to volume ratio of CdSe tetrapods compared to CdSe dots may be also responsible for reduced charge trapping features in the P3HT/CdSe tetrapod system. Since CdSe tetrapods have a significantly reduced surface to volume ratio compared to CdSe dots (calculated to be $\sim 50 \%$ lower), for the two P3HT/CdSe nanocrystal devices with the same weight ratio and also the 
similar quantum confinement of the CdSe particles, the P3HT/ CdSe dot device will necessarily possess a total nanocrystal surface area that is twice as much as the P3HT/CdSe tetrapod device. Therefore, the reduced total interfacial area in the $\mathrm{P} 3 \mathrm{HT} / \mathrm{CdSe}$ tetrapod system results in a lower density of interfacial trap states that may be responsible for reduced charge trapping and hence less severe SRH/trap-mediated recombination compared to the P3HT/CdSe dot system. Furthermore, a reduced voltage dependence of in the $\mathrm{P} 3 \mathrm{HT} / \mathrm{CdSe}$ tetrapod system may suggest shallower interfacial trap states in the P3HT/CdSe tetrapod system compared to the P3HT/CdSe dot system. Finally, the pronounced shoulder at $\sim 600 \mathrm{~nm}$ of the EQE spectrum may also facilitate enhanced transport of holes in the $\mathrm{P} 3 \mathrm{HT} / \mathrm{CdSe}$ tetrapod device. It is reported by previous studies $^{22}$ that P3HT is crystallised into a fibrillar nanostructures resulting in enhanced hole transport in the $\mathrm{P} 3 \mathrm{HT} /$ CdSe nano-rods system. Furthermore, the larger physical size of the CdSe tetrapods is more likely to result in better interconnection of the P3HT phase. Therefore, improved properties of the P3HT phase may also contribute to the improved charge collection properties of the P3HT/CdSe tetrapod system. Nevertheless, while nanoparticle shape is an important factor reported herein, it should be noted that there are other factors (for example the differences in nano-morphology) that might contribute to the differences in charge transport and recombination kinetics of polymer/nanocrystal solar cells, which is beyond the scope of this paper.

\section{Experimental}

\section{Materials preparation}

P3HT was supplied by Merck Inc. (molecular weight $M_{\mathrm{w}} \sim$ $54 \mathrm{~kg} \mathrm{Mol}{ }^{-1}$, regioregularity RR 94\%) and was used as-received. CdSe nano-dot synthesis followed the double hot-injection method developed by Yu and Peng. ${ }^{26}$ Two Se precursors were separately prepared by mixing $0.345 \mathrm{~g}$ of selenium powder and $3.825 \mathrm{~g}$ of trioctylphosphine (TOP) and heating at $80{ }^{\circ} \mathrm{C}$ for one hour with magnetic stirring. The $\mathrm{Cd}$ precursor was prepared by mixing $0.748 \mathrm{~g}$ of cadmium oxide (CdO) powder with $15 \mathrm{~g}$ of 1-octadecene (ODE) and $25 \mathrm{~g}$ of oleic acid (OA) in a $250 \mathrm{~mL}$ threeneck flask. The mixture was then heated to $230{ }^{\circ} \mathrm{C}$ under nitrogen atmosphere with magnetic stirring till a colourless solution was formed. Then the first Se precursor was rapidly injected into the Cd precursor solution, allowing for the formation and the growth of the nanocrystals. The second Se precursor was injected into the solution 15 minutes after the first injection for further growth of the nanocrystals. The reaction was stopped by rapidly cooling down the flask to room temperature in cold water 45 min after the second injection. The nanocrystals were recovered by centrifugation in methanol at $3000 \mathrm{rpm}$ for $40 \mathrm{~min}$ and washed three times to remove excessive reactants and impurities, followed by dissolving in $15 \mathrm{~mL}$ of $n$-butylamine for ligand exchange. For CdSe nano-tetrapod synthesis, the Se precursor was prepared by mixing $73 \mathrm{mg}$ of Se and $1916 \mathrm{mg}$ of tributylphosphine (TBP) followed by heating at $90{ }^{\circ} \mathrm{C}$ under $\mathrm{N}_{2}$ with magnetic stir, until a clear solution was obtained. The Cd precursor was prepared by mixing $400 \mathrm{mg}$ of CdO, $320 \mathrm{mg}$ of hexylphosphonic acid (HPA), $1420 \mathrm{mg}$ of 1-tetradecylphosphonic acid (TDPA) and $3000 \mathrm{mg}$ of trioctylphosphine oxide (TOPO) in a $50 \mathrm{ml}$ three-neck-flask followed by degassing at $120{ }^{\circ} \mathrm{C}$ for $1 \mathrm{~h}$. After heating to $300{ }^{\circ} \mathrm{C}$ for 5 min under $\mathrm{N}_{2}$, the Cd precursor was cooled down to room temperature and aged for $48 \mathrm{~h}$. After refrigerating at $5{ }^{\circ} \mathrm{C}$, the Se precursor was rapidly injected into the Cd precursor at $300{ }^{\circ} \mathrm{C}$. The growth of the nanocrystals was allowed for $5 \mathrm{~min}$ and quenched by $5 \mathrm{ml}$ toluene. Finally the tetrapods were precipitated with propan-2-ol and washed with toluene.

\section{Device preparation}

For device preparation, glass substrates coated with a patterned ITO stripe were cleaned by sonicating in acetone and isopropanol successively, followed by oxygen plasma treatment. A $40 \mathrm{~nm}$ thick PEDOT:PSS layer was deposited onto the plasma-treated substrates followed by heating at $150{ }^{\circ} \mathrm{C}$ for 30 minutes under flowing nitrogen. The substrates were then transferred into a nitrogen glovebox for further fabrication steps. P3HT and CdSe nanoparticles were separately dissolved in anhydrous chlorobenzene and anhydrous chloroform respectively, each with a solution concentration of $30 \mathrm{mg} \mathrm{ml}^{-1}$. These solutions were then mixed with a volume ratio of $1: 9$ to form a blend solution with an optimized weight ratio of 1:9 P3HT: CdSe ( 1:2 P3HT: CdSe by volume). Devices made with other weight ratios always exhibit lower power efficiencies. This mixed solution was spin-coated at a spin speed of $2000 \mathrm{rpm}$ to form $100 \mathrm{~nm}$ thick films. Device fabrication was completed by pre-annealing of the films at $150{ }^{\circ} \mathrm{C}$ for 30 minutes followed by thermal evaporation of $100 \mathrm{~nm}$ thick aluminium electrode under high vacuum (less than $10^{-6}$ mbar) through an 8-pixel shadow mask. Over 12 devices were fabricated for both system, and 3 typical devices with average performance were selected for device physics studies through out this paper to ensure a fair comparison.

\section{Device characterisation}

External quantum efficiency (EQE) spectra of devices were measured with a $100 \mathrm{~W}$ tungsten halogen lamp dispersed through a monochromator with a Keithley 237 source meter unit (SMU) used to measure the short-circuit current as a function of wavelength. Incident light intensity was monitored during measurement by splitting of part of the beam to reference photodiode to account for intensity fluctuations. The system was calibrated using a Hamamatsu S8746-01 calibrated photodiode at the sample position. Current-voltage $(J-V)$ characteristics were measured under simulated AM 1.5G conditions using an Oriel 81160-1000 solar simulator, at an intensity equivalent to $100 \mathrm{~mW} \mathrm{~cm}{ }^{-2}$ after correcting for spectral mismatch.

\section{Transient photocurrent and photovoltage measurements}

The transient photocurrent and transient photovoltage methodology employed herein followed a protocol described in previous publications. $^{8,20}$ Square light pulses of $200 \mu$ s duration were generated by a high-brightness $525 \mathrm{~nm}$ green LED (Kingbright, L-7104VGC-H) powered by a Hewlett Packard (HP) 8116A 
pulse/function generator. The rise and fall times of the LED were measured to be less than 50 ns by a New Focus 1621 ns photodetector. A neutral density filter wheel was used to vary the pulse intensity and a BPX-65 silicon solar cell was used as the reference cell for light intensity calibration. Short-circuit photocurrent transients were recorded by connecting the device under test in series with an Agilent DSO6052A digitising oscilloscope with input impedance of $50 \Omega$. For voltage-dependent transient photocurrent measurements, devices were connected in series with another HP 8116A pulse/function generator operating in DC mode with current measured using the $50 \Omega$ input impedance of the oscilloscope. Care was taken to make sure that the photocurrent transients recorded were not RC-limited, using methods previously described. ${ }^{8}$ Transient photovoltage measurements were performed using a similar setup to the photocurrent measurements with the device under test connected to the oscilloscope with a $1 \mathrm{M} \Omega$ input impedance. Background illumination was provided by a high-brightness white ring LED array. The light intensity of the pulsed light was kept sufficiently low such that the voltage perturbation was always less than $5 \%$ of the open-circuit voltage created by the constant background illumination.

\section{Conclusions}

We have presented a direct side-by-side comparison of the transient photocurrent and transient photovoltage behaviour of two P3HT/CdSe nanoparticle devices, probing the influence of the nanoparticle shape on the device operation of this benchmark hybrid system. By investigating the light intensity and voltage dependence of the rise and decay kinetics of the photocurrent transients in response to a $200 \mu$ s square light pulse, significant differences in the device operations for these efficient polymer/nanocrystal systems are revealed. For the P3HT/CdSe nano-dots system, the photocurrent transients are characterised by an initial transient photocurrent peak the magnitude of which becomes more pronounced with increasing applied bias voltage, indicative of Shockley-Read-Hall (SRH) and/or trapmediated recombination caused by the build-up of the trapped charge carriers. After turn-off, the observation of a long photocurrent tail is consistent with the slow detrapping process of trapped charges. The similarity of the extracted charge $v s$. applied bias voltage and the measured $J-V$ characteristics suggests that the charge collection of the P3HT/CdSe nano-dot device is limited by severe charge trapping in this system. In contrast, the P3HT/CdSe nano-tetrapods system exhibits significantly reduced charge trapping, evidenced by the absence of the initial transient photocurrent peak even at short-circuit, less severe back injection of charge carriers following turn-off, voltage-dependent photocurrent decay transients and a relatively constant extracted charge vs. applied bias voltage. Transient photovoltage measurements also reveal much longer charge carrier lifetimes and less severe non-geminate recombination losses in the P3HT/CdSe tetrapod device compared to the $\mathrm{P} 3 \mathrm{HT} /$ CdSe dot device under constant illumination at open circuit.
These direct observations reveal the shape of the nanoparticles as a critical factor influencing charge transport in this benchmark hybrid system, confirming the previous hypothesis that the use of tetrapods improves device performance through an improvement in electron transport in the nanocrystal phase. Our findings also highlight that reducing the severity of charge trapping through engineering of the nanocrystal phase is an effective strategy to improve the performance of hybrid solar cells.

\section{Acknowledgements}

This work was supported by the Engineering and Physical and Sciences Research Council, U.K. (Supergen Excitonic Solar Cell Consortium, EP/G031088/1 IV, Advanced Research Fellowship, EP/E051804/1). Z.L. thanks the Cambridge Overseas Trust and the Mott Physics of the Environment Award for financial support.

\section{Notes and references}

1 Z. He, C. Zhong, S. Su, M. Xu, H. Wu and Y. Cao, Nat. Photonics, 2012, 6, 591-595.

2 C. H. Peters, I. T. Sachs-Quintana, J. P. Kastrop, S. Beaupré, M. Leclerc and M. D. McGehee, Adv. Energy Mater., 2011, 1, 491-494.

3 S. Ren, L.-Y. Chang, S.-K. Lim, J. Zhao, M. Smith, N. Zhao, V. Bulović, M. Bawendi and S. Gradečak, Nano Lett., 2011, 11, 3998-4002.

4 T. $\mathrm{Xu}$ and Q. Qiao, Energy Environ. Sci., 2011, 4, 2700-2720.

5 N. C. Greenham, X. Peng and A. P. Alivisatos, Phys. Rev. B: Condens. Matter Mater. Phys., 1996, 54, 17628-17637.

6 Y. Zhou, F. S. Riehle, Y. Yuan, H.-F. Schleiermacher, M. Niggemann, G. A. Urban and M. Krüger, Appl. Phys. Lett., 2010, 96, 013304.

7 M. D. Heinemann, K. von Maydell, F. Zutz, J. Kolny-Olesiak, H. Borchert, I. Riedel and J. Parisi, Adv. Funct. Mater., 2009, 19, 3788-3795.

8 Z. Li, F. Gao, N. C. Greenham and C. R. McNeill, Adv. Funct. Mater., 2011, 21, 1419-1431.

9 Z. Chen, H. Zhang, W. Yu, Z. Li, J. Hou, H. Wei and B. Yang, Adv. Energy Mater., 2013, 3, 433-437.

10 F. Gao, S. Ren and J. Wang, Energy Environ. Sci., 2013, 6, 2020-2040.

11 W. U. Huynh, X. Peng and A. P. Alivisatos, Adv. Mater., 1999, 11, 923-927.

12 J. C. Hindson, Z. Saghi, J.-C. Hernandez-Garrido, P. A. Midgley and N. C. Greenham, Nano Lett., 2011, 11, 904-909.

13 B. Sun, H. J. Snaith, A. S. Dhoot, S. Westenhoff and N. C. Greenham, J. Appl. Phys., 2005, 97, 014914-014916.

14 S. Dayal, N. Kopidakis, D. C. Olson, D. S. Ginley and G. Rumbles, Nano Lett., 2009, 10, 239-242. 
15 B. Sun, E. Marx and N. C. Greenham, Nano Lett., 2003, 3, 961-963.

16 H.-C. Chen, C.-W. Lai, I. C. Wu, H.-R. Pan, I. W. P. Chen, Y.-K. Peng, C.-L. Liu, C.-h. Chen and P.-T. Chou, Adv. Mater., 2011, 23, 5451-5455.

17 E. Martínez-Ferrero, J. Albero and E. Palomares, J. Phys. Chem. Lett., 2010, 1, 3039-3045.

18 W. U. Huynh, J. J. Dittmer, N. Teclemariam, D. J. Milliron, A. P. Alivisatos and K. W. J. Barnham, Phys. Rev. B: Condens. Matter Mater. Phys., 2003, 67, 115326.

19 S. Dayal, M. O. Reese, A. J. Ferguson, D. S. Ginley, G. Rumbles and N. Kopidakis, Adv. Funct. Mater., 2010, 20, 2629-2635.

20 C. R. McNeill, I. Hwang and N. C. Greenham, J. Appl. Phys., 2009, 106, 024507-024508.
21 A. Maurano, R. Hamilton, C. G. Shuttle, A. M. Ballantyne, J. Nelson, B. O'Regan, W. Zhang, I. McCulloch, H. Azimi, M. Morana, C. J. Brabec and J. R. Durrant, Adv. Mater., 2010, 22, 4987-4992.

22 B. Sun and N. C. Greenham, Phys. Chem. Chem. Phys., 2006, 8, 3557-3560.

23 I. Hwang, C. R. McNeill and N. C. Greenham, J. Appl. Phys., 2009, 106, 094506-094510.

24 Z. Li, G. Lakhwani, N. C. Greenham and C. R. McNeill, J. Appl. Phys., 2013, 114, 034502.

25 L. J. A. Koster, O. Stenzel, S. D. Oosterhout, M. M. Wienk, V. Schmidt and R. A. J. Janssen, Adv. Energy Mater., 2013, 3, 615-621.

26 W. W. Yu and X. Peng, Angew. Chem., Int. Ed., 2002, 41, 2368-2371. 